\title{
Tetrapterys Cav. (Malpighiaceae) from Brazilian Midwest
}

\author{
Augusto Francener ${ }^{1}$, Geraldo Alves Damasceno-Júnior ${ }^{2}$ and Vera Lúcia Gomes-Klein ${ }^{3}$
}

Received: June 18, 2014. Accepted: October 30, 2014

\begin{abstract}
We present a floristic survey of the genus Tetrapterys (Malpighiaceae) from the Brazilian Midwest, including morphological descriptions, keys, comments on taxonomy and phenology, illustrations, and distribution maps for all species. Nine species were found in the studied area: T. ambigua, T. crispa, T. discolor, T. hassleriana, T. jussieuana, T. microphylla, T. mucronata, T. racemulosa, and T. ramiflora. The distribution of species shows a collection gap mostly in northern States of Mato Grosso and southeastern Mato Grosso do Sul. Only T. ambigua and T. ramiflora were found in all federal units within the Brazilian Midwest, and three species were recorded for just one, T. hassleriana and T. racemulosa in Mato Grosso do Sul, and T. microphylla in Goiás, restricted to the Chapada dos Veadeiros.
\end{abstract}

Keywords: Cerrado, Floristic Survey, Malpighiaceae, Taxonomy, Tetrapterys

\section{Introduction}

Malpighiaceae is a monophyletic family of flowering plants comprising approximately 77 genera and 1300 species distributed within the tropics (Cameron et al. 2001, Davis \& Anderson 2010), of which $85 \%$ are restricted to the Neotropics (Davis et al. 2001). Its species are commonly found within open habitats, such as savannas, gallery forests, and rainforests, with a few species occupying xeric habitats (Anderson 1979).

Tetrapterys is a genus of Malpighiaceae comprising 69 species (Anderson et al. 2006), occurring from Mexico to Argentina (Anderson 1998). They are recognized by sepals not covering the floral bud before anthesis and a schizocarpic fruit splitting into three samaroid mericarps with lateral wings in the shape of an "X," often with a reduced dorsal wing (Mamede 1987; Anderson 2006). In Brazil, 25 species have been found, of which 16 are regarded as endemic (Mamede 2014).

The genus is paraphyletic, being divided into two wellsupported clades belonging to an informal group called Tetrapteroids. One of the clades is the sister-group of Heteropterys and mostly comprises climbing species from forest edges, while the other clade is the sister-group of Niedenzuella and comprises shrubs to subshrubs from open habitats, such as the Cerrado (Davis \& Anderson 2010).
Niedenzuella includes 16 species previously regarded as belonging to a section of Tetrapterys, but differing from it by having sepals longer than petals on the floral bud during anthesis (Anderson 2006).

The group has been the subject of just a few taxonomic studies in Brazil, mostly floristic surveys of Malpighiaceae and general checklists. Among studies regarding species of Tetrapterys (in a broad sense, including Niedenzuella) in Brazil, we can highlight the surveys undertaken at Fontes do Ipiranga State Park (SP) of two species (Mamede 1984), at Serra do Cipó (MG) of five species (Mamede 1987), and at Ducke Reserve (AM) of two species (Vicentini \& Anderson 1999). Within checklists, we can highlight the studies at States of Mato Grosso and Mato Grosso do Sul of nine species of Tetrapterys (Anderson 1998), at Cerrado Biome of 15 species (Mendonça et al. 2008), and at Federal District of six species (Cavalcanti \& Ramos 2001).

Due to the few studies conducted for the group in Brazil, it was important to undertake a survey of Tetrapterys from the Brazilian Midwest. This poorly known floristic region, including $18.2 \%$ of national territory (IBGE 2009), only has knowledge about the genus in the form of checklists. Thus, the goal of this study was to perform a floristic survey and to present the species distribution of Tetrapterys from the Brazilian Midwest region.

\footnotetext{
${ }_{1}^{1}$ Aluno do Programa de Pós-graduação em Biologia Vegetal/UFMS; Av. Senador Filinto Müller s/n - Cidade Universitária 79070-900 - Campo Grande, MS - Brasil. augustofng@yahoo.com.br

${ }^{2}$ Professor do Programa de Pós-graduação em Biologia Vegetal/UFMS; Av. Senador Filinto Müller s/n - Cidade Universitária 79070-900 - Campo Grande, MS - Brasil ${ }^{3}$ Professora Associada III Universidade Federal de Goiás; Departamento de Botânica, Campus Samambaia 74001-970 - Goiânia, GO - Brasil - Caixa-Postal: 131
} 


\section{Material and methods}

We performed 13 field expeditions in order to collect specimens, capture photographs, and observe populations of Tetrapterys Cav. in their natural habitat. Field collections were conducted in different regions of the States of Mato Grosso do Sul, Mato Grosso, and Goiás, collecting specimens with flowers and/or fruits. All collected specimens were preserved according to standard botanical techniques (Bridson \& Forman 1992), and vouchers were incorporated into the CGMS herbarium, with duplicates sent to UFMT and UFG herbaria (acronyms are defined according to Thiers 2014).

Herbaria holding collections from the Brazilian Midwest were consulted (CEN, CGMS, COR, CPAP, HERBAM, HAPN, HEPH, IBGE, NX, UB, UFMT, and UFG), in addition to herbaria holding important collections of Tetrapterys from the studied area that would add relevant information to the study (INPA, HUEFS, MBM, RB, R, SP, and USZ).

We elaborated on standardized descriptions for each species and made additional comments, including information about habitat, phenology, taxonomic comments, and geographic distribution. Maps were elaborated using Diva software, and specimens without geographic coordinates were represented by their locality coordinate retrieved from SpeciesLink (2010).

Morphological descriptions were based on Radford et al. (1974). Inflorescence descriptions were based on Weberling (1989) and Anderson (1981), and the remaining characters were based on traditional terminology used for Malpighiaceae morphology. Descriptions of pedicels were based on Anderson (1981), Mamede (1990), and Anderson (1997); trichome morphology was based on Gates (1982). Within species descriptions, we described hair morphology by shape $[(\mathrm{Y}),(\mathrm{T})$ or $(\mathrm{V})]$ and conducted measurements using a stereoscopic microscope STUDARlab with a micrometric barrel Me Opta. Valid names and synonyms were verified in Anderson et al. (2006) and authors names in Brummitt \& Powel (1992).

We also elaborated a key and illustrations for all species found in the studied area, providing detailed evidence for their distribution, habit, leaves, inflorescence, flowers, fruits, and structures to help species identification. Species not illustrated here can be found in Niedenzu (1928).

\section{Results and discussion}

Tetrapterys Cav. in Monadelphiae Classis Dissertationes Decem9: 433, t. 260.1790.

Type: Tetrapterys inaequalis Cav.

Subshrubs, shrubs, and lianas. Leaves opposite, rarely subopposite or verticillate; laminas lanceolate to obovate, margin plane or revolute, glands at one or both sides, on the margin or between primary vein and margin; petioles glandular or eglandular; stipules interpetiolar or intrapetiolar. Inflorescence simple, racemose terminating in umbels or cincinii, sessile to pedunculate; pedicels pedunculate or sessile; foliose bracts; bracts, bracteoles, and peduncle glandular or eglandular. Sepals five, connate, glabrous, or sericeous, hairs brownish; glands (when present) disposed in pairs at each sepal, usually absent on the anterior sepal, adpressed or stalked. Floral buds orange to yellowish, with sepals not covering petals before anthesis. Petals five free (one posterior and four lateral), unguiculate, yellow, posterior glabrous, margin entire or indented. Stamens ten, connate at base. Ovary tricarpellate, trilocular, glabrous, sericeous or tomentose, lateral wings usually present; styles three, erect, slightly lyrate outwards; styles alike, subequal or anterior style longer than the posterior ones; stigma apical, slightly bent towards the center of the flower. Samaroid mericarps with two to four lateral wings, dorsal wing smaller or as long as the lateral ones, sometimes with outgrowths between the lateral and dorsal wings.

The Brazilian Midwest is a region with few collections of Malpighiaceae, mostly from the States of Mato Grosso and Mato Grosso do Sul (WR Anderson unpubl. res.). Nine species of Tetrapterys have been found: T. ambigua, T. crispa, T. discolor, T. hassleriana, T. jussieuana, T. microphylla, T. mucronata, T. racemulosa and T. ramiflora. Besides these species, T. chamaecerasifolia, T. phlomoides and T. salicifolia have also been cited for the Midwest (Mamede 2014). However, there are no specimens to confirm their occurrence within the studied area, and the records are represented by misidentified specimens. Only four species were found in the field and this is related to the difficulty of finding these species in their natural habitat because they are not abundant. Consequently, some species are only represented in old collections.

\section{Key for species of Tetrapterys from the Brazilian Midwest}

1. Shrub, subshurub or scandent subshrub; stipules intrapetiolar; foliar laminas with glands on the margin; inflorescence simple racemose or a raceme of cincinii.

2. Inflorescence a raceme of cincinii, sessile, congested at stem (cauliflorous)

9. T. ramiflora

2. Inflorescence a simple raceme, pedunculate, lax

3. Samaroid mericarps with two or four lateral wings, similar to the dorsal wing

4. Samaroid mericarps with four lateral wings

8. . racemulosa

4. Samaroid mericarps with two lateral wings

5. Foliar lamina villous to lanose, on both sides; hairs with base up to $0.2 \mathrm{~mm}$ long and branches up to 2.0 $\mathrm{mm}$ long. 
5. Foliar lamina sericeous abaxially and slightly scabrous adaxially; hairs with base up to $0.1 \mathrm{~mm}$ long and branches up to $1.2 \mathrm{~mm}$ long..... 5. T. jussieuana

3. Samaroid mericarps with four lateral wings differing from the dorsal wing.

6. Shrubs; abaxial surface of lamina sericeous; restricted to highland cerrado rupestre and campos rupestres 6. T. microphylla

6. Scandent subshrub; abaxial surface of lamina glabrescent; restricted to lowland flooded fields in the Pantanal and Araguaia River. 4. T. hassleriana

1. Liana; stipules interpetiolar, foliar lamina with glands between the primary vein and the margin; inflorescence racemose ending in umbels.

7. Stipules free; foliose bracts lanceolate; sepals with glands stalked, auriculate or absent. 7. T. mucronata

7. Stipules connate; foliose bracts obovate to widely elliptic; sepals with glands adpressed, elongate.

8. Petiole glabrous; samaroid mericarps with outgrowths between dorsal and lateral wings. 3. T. discolor

8. Petiole sericeous; samaroid mericarps without outgrowths between dorsal and lateral wings 2. T. crispa

\section{Species descriptions}

1. Tetrapterys ambigua (A.Juss.) Nied. in Das Pflanzenreich 141: 168. 1928.

Figs. 1 and $2 \mathrm{~A}-\mathrm{H}$

Subshrub 0.1-0.6 m, stem villous to lanose (T). Leaves opposite, rarely subopposite or verticillate; foliar lamina $1.8-8.5 \mathrm{~cm}$ long, $0.8-3.8 \mathrm{~cm}$ wide, narrow-elliptic, elliptic to obovate, base cuneate, apex acute, retuse to slightly cuspidate, margin plane to slightly revolute, with glands on the margin at base, villous to lanose on both sides, hairs long, base up to $0.2 \mathrm{~mm}$ long and branches up to $2.0 \mathrm{~mm}$ long (T); petiole 1.5-5.0 $\mathrm{mm}$ long, villous to lanose $(\mathrm{T})$, glands rarely disposed on the apex; stipules $0.7-1.3 \mathrm{~mm}$ long, intrapetiolar, triangular. Inflorescence a simple raceme, lax; bracts $2.2-3.3 \mathrm{~mm}$, elliptic; peduncles $1.2-10.0 \mathrm{~mm}$ long, villous to lanose (T); bracteoles $2.3-2.6 \mathrm{~mm}$ long, glandular at base or eglandular; pedicels $2.3-8.0 \mathrm{~mm}$ long, villous to lanose (T). Sepals $2.7-3.3 \mathrm{~mm}$ long, $1.5-2.3 \mathrm{~mm}$ wide, ovate, sericeous (T), with $8-9$ glands $1.8-2.3 \mathrm{~mm}$ long, $1.1-1.3 \mathrm{~mm}$ wide, adpressed elongated. Floral buds orange to yellowish. Petals slightly indented at margin, sometimes main veins orange, lateral petals, limb $6.0-6.7 \mathrm{~mm}$ long, 4.2-5.5 mm wide, claw $1.5-2.0 \mathrm{~mm}$ long, posterior petal, limb 5.0-6.7 mm long., 3.0-4.5 mm wide, claw $2.0-2.2 \mathrm{~mm}$ long. Stamens heteromorphic; filaments $2.2-2.6 \mathrm{~mm}$ long; anthers $1.0-1.2 \mathrm{~mm}$ long. Ovary $0.9-1.5 \mathrm{~mm}$ long, villous to lanose ( $\mathrm{T}$ and $\mathrm{V}$ ), lateral wings present; styles $2.5-3.0 \mathrm{~mm}$ long, alike. Samaroid mericarps with two lateral wings, alike dorsal wing, glabrous, lateral wings 6.0-9.0 long, 15.0-19.0 $\mathrm{mm}$ wide, dorsal wing $7.0-10.0 \mathrm{~mm}$ long, $13.0-16.0 \mathrm{~mm}$ wide; nut villous to lanose ( $\mathrm{V}$ and $\mathrm{Y}$ ).

Specimens examined: BRAZIL. Distrito Federal: Brasília, 7/XI/2002, fl., A.A. Santos et al. 1603 (IBGE); 6/ IX/1960, fl., A.G. Andrade \& M. Emmerich 410, 402 (R); 29/IX/2001, fl., G. Pereira-Silva 5474 (CEN); 3/VIII/1976, fl., J.A. Ratter et al. 3347 (UB); 12/VIII/1980, fl., L. Fiedler 111 (MBM); 3/X/2002, fl. fr., M. Aparecida da Silva 5395 (IBGE, UB); 29/VII/2008, fl. fr., S.C. Cappellari 2280 (IBGE,
UB); 14/X/2003, fr., V.F. Paiva \& L.C. Milhomens 155 (HEPH). Planaltina, 28/IX/1994, fr., J.C. Silva 752 (UB). Goiás: Alto Paraíso de Goiás, 15/X/2010, fl., A. Francener et al. 1012 (CGMS, UFG); 15/X/2010, fr., A. Francener et al. 1023 (CGMS, UFG); 19/IV/1998, fl., H.D. Ferreira 3562 (UFG); 7/X/1972, fr., J.A. Rizzo 8451 (UFG). Aparecida de Goiânia, 1/XII/2002, fl. fr., J.F.B. Pastore 36 (CEN). BR-020, 17/VIII/1990, fl. T.B. Cavalcanti et al. 813 (CEN). Campinaçu, 6/VII/1995, fl., B.M.T. Walter et al. 2669 (CEN). Campos Belos, 22/X/2001, fl. fr., M.L. Fonseca et al. 3019 (IBGE). Cavalcante, 9/XI/2000, fr., B.M.T. Walter et al. 4656 (CEN). Corumbá de Goiás, 18/IV/1996, fl., H.D. Ferreira 3593 (UFG). Jataí, 20/IX/1973, fl., J. A. Rizzo 9299 (UFG). Luziânia, 1/IV/1984, fl., F.B. Magalhães 4 (UB). Minaçu, 9/X/1991, fl., T.B. Cavalcanti et al. 877 (CEN). Mineiros, 14/X/1995, fl., J.P. Oliveira 207 (UFG). Mossâmedes, 17/ VI/1994, fl. fr., J. A. Rizzo et al. 11481 (UFG). Niquelândia, 13/VIII/1996, fl., M.L. Fonseca et al. 1079 (IBGE); 18/X/1996, fr., R. Marquete et al. 2662 (IBGE); 20/X/1996, fl., R. Marquete et al. 2715 (IBGE); 13/VIII/1996, fr. R.C. Mendonça et al. 2542 (IBGE). Padre Bernardo, 21/IX/1972, fl. fr., J.A. Ratter et al. 2462 (UB). Piracanjuba, 26/IX/1975, fl. fr., G. Hatschbach 37155 (MBM). São João da Aliança, 30/X/1979, fr., E.P. Heringer et al. 2634 (IBGE). Mato Grosso: $300 \mathrm{Km}$ from Cuiabá to Porto Velho, 28/IX/1963, fl., J.M. Pires 56908 (UB). Água Boa, 16/IX/1984, fl., L. Coradin et al. 7320 (CEN). Alto Araguaia, 22/IX/1988, fl. fr., R. Kral \& M.G.L. Wanderley 75016 (SP). Alto Paraguai, 23/X/1995, fl., B. Dubs 1897 (UFMT). Barra do Garças, 15/IX/1968, fl., G. Eiten \& L.T. Eiten 8755 (SP). Cuiabá, 30/IX/1980, fl., A.L. Prado 186 (UFMT); 21/X/1980, fr., A.L. Prado 207 (UFMT); 30/IX/1977, fl. fr., M. Macedo et al. 982 (UFMT). Garapú, 2/X/1964, fl., H.S. Irwin \& T.R. Soderstrom 6606 (R). Nova Xavantina, 6/V/2001, fl., G.P. Freitas 55 (NX); 10/X/2001, fl., T.M.E.S. Pinto 9 (IBGE, NX). Novo Santo Antônio, 30/X/2005, fr., M.C. Moresco et al. 115 (NX, SP). Primavera do Leste, 6/X/1988 fl., R. Kral \& M.G.L. Wanderley 75169 (SP). Mato Grosso do Sul: Serra do Rio Preto, 18/XI/1965, fr., H.S. Irwin et al. 10456 (UB). Bela Vista, 23/X/1987, fr., G. Hatschbach. \& J.M. Silva 51576 (MBM). Corumbá, 

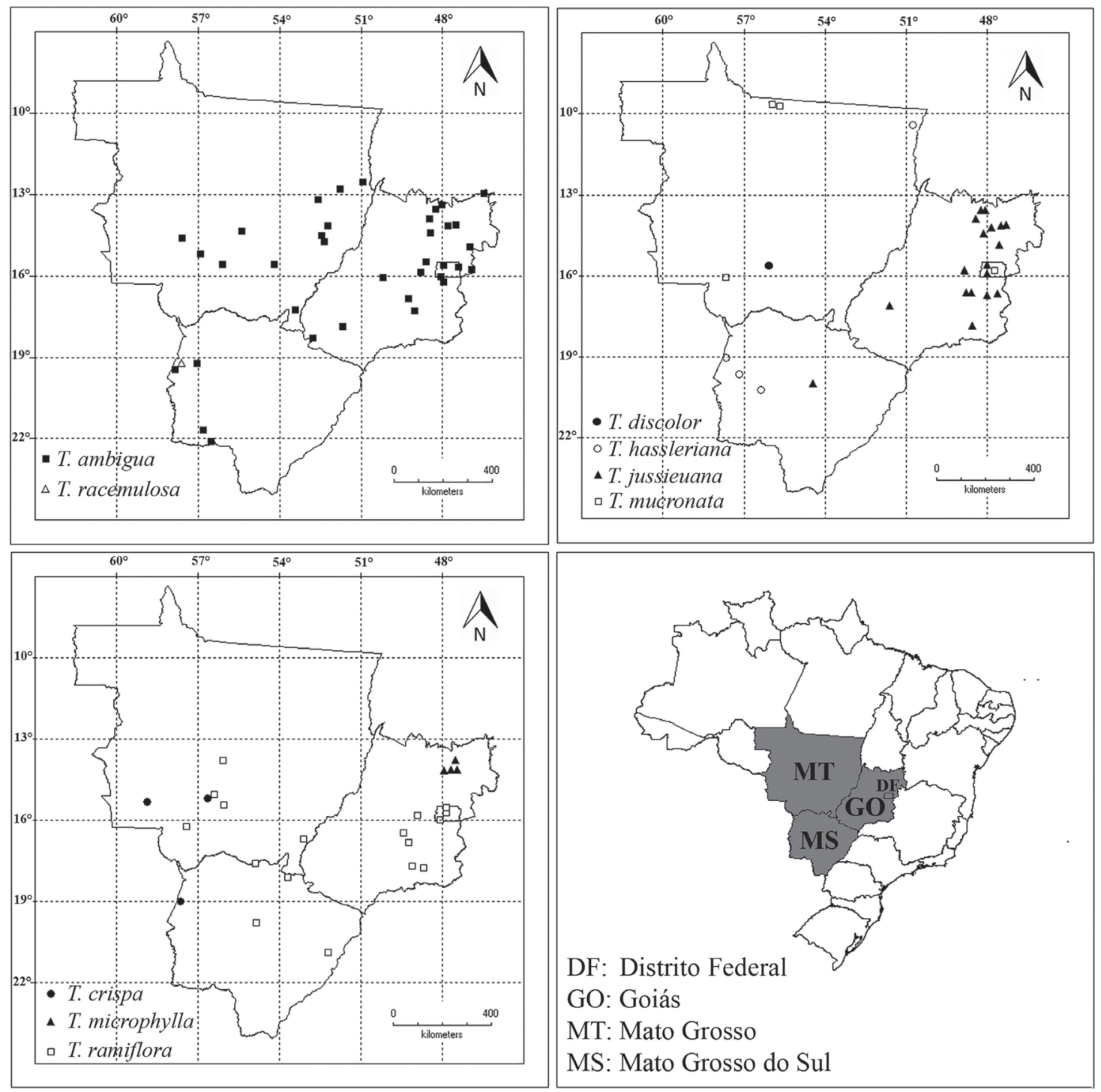

Figure 1. Distribution map of Tetrapterys species from the Brazilian Midwest.

09/X/1995, fl., G.A. Damasceno-Júnior et al. 924 (SP); 10/ XI/1995, fr., I.M. Bortolotto 115 (SP); 24/X/1988, fl., M.L. Gasparini 45 (CPAP, SP). Porto Murtinho, 26/IX/1996, fr., J.A. Ratter et al. 7552 (UB).

Tetrapterys ambigua is common in Cerrado sensu lato areas in Brazil, extending to Bolivia, flowering mostly after fires during the dry season. It shows an underground xylopodium, which allows it to resprout after fires. The only similar species is T. jussieuana, differing mostly on leaf indumentum. T. ambigua shows a foliar lamina villous to lanose ( $\mathrm{T}$ ), hairs with base and branches long (bases up to $0.2 \mathrm{~mm}$ long and branches up to $2.0 \mathrm{~mm}$ long), whereas T. jussieuana shows a foliar lamina sericeous (T) abaxially, and hairs with base and branches short (bases up to $0.1 \mathrm{~mm}$ long and branches up to $1.2 \mathrm{~mm}$ long). In the Midwest, T. T. ambigua flowers from April to December and fruits from May to December.
2. Tetrapterys crispa A.Juss. in Ann. Sci. Nat. 2 Ser. Bot. 13: 265.1840.

Figs. 1 and $3 \mathrm{E}-\mathrm{H}$

Liana, stem glabrous or pubescent $(\mathrm{T})$ on branches near the inflorescence. Leaves opposite; foliar lamina 6.2-8.3 $\mathrm{cm}$ long, 3.0-4.0 cm wide, elliptic, base subcordate, apex acuminate, margin plane, with glands between primary vein and margin on both sides up to the middle of the lamina, abaxial surface pubescent $(\mathrm{T})$ and sericeous $(\mathrm{T})$ on primary vein, adaxial surface pubescent $(\mathrm{T})$, mostly near veins; petiole $7.0-10.0 \mathrm{~mm}$ long, sericeous $(\mathrm{T})$, eglandular; stipules 1.2-2.1 mm long, interpetiolar, connate, triangular, deciduous, most nodes retain a scar. Inflorescence a raceme ending in umbels; foliose bracts $9.0-18.0 \mathrm{~mm}$ long, $8.0-12.0$ $\mathrm{mm}$ wide, obovate to widely elliptic; bracts approximately $2.0 \mathrm{~mm}$, lanceolate; peduncle $2.5-4.0 \mathrm{~mm}$ long, tomentose 
(T); bracteoles 1.8-2.0 mm long, eglandular; pedicel 3.2-4.0 $\mathrm{mm}$ long, tomentose (T). Sepals $2.0-2.7 \mathrm{~mm}$ long, $1.5-1.8$ $\mathrm{mm}$ wide, rounded, glabrous, 8-glandular, glands 2.5-3.8 $\mathrm{mm}$ long, $1.0-1.7 \mathrm{~mm}$ wide, adpressed, elongate. Floral buds yellow. Petals entire at margin, lateral petals, limb 5.0-8.0 mm long, 4.5-6.0 $\mathrm{mm}$ wide, claw $0.9-1.2 \mathrm{~mm}$ long, posterior petal, limb $5.0-5.1 \mathrm{~mm}$ long, $3.5-3.6 \mathrm{~mm}$ wide, claw 1.5-2.0 mm long. Stamens heteromorphic; filaments $1.8-2.5 \mathrm{~mm}$ long; anthers $1.1-1.3 \mathrm{~mm}$ long. Ovary 1.4-1.6 $\mathrm{mm}$ long, sericeous (T), lateral wings present; styles 2.6-2.9 $\mathrm{mm}$ long, subequal. Samaroid mericarps with four lateral wings, differing from the dorsal wing, pubescent $(\mathrm{V})$, superior lateral wings $17.0-20.0 \mathrm{~mm}$ long, $7.0-8.5 \mathrm{~mm}$ wide, inferior lateral wings $8.0-10.0 \mathrm{~mm}$ long, $4.0-5.5 \mathrm{~mm}$ wide, dorsal wing 3.0-5.0 $\mathrm{mm}$ long, 6.0-9.0 $\mathrm{mm}$ wide; outgrowths between dorsal and lateral wings absent; nut floccose (V).

Specimens examined: BRAZIL. Mato Grosso: Jauru. no date, fl., G. Hatschbach et al. 62456 (HUEFS); 8/V/1995, fl., G. Hatschbach et al. 62481 (MBM). Serra das Araras, 1/ IV/1996, fl., B. Dubs 2159 (UFMT). Mato Grosso do Sul: Corumbá, 14/III/1990, fr., L.F. Boabaid 2 (SP).

Tetrapterys crispa occurs on forest edges, from South Brazil to northern South America, extending to southeast and northeast Brazil. Shows interpetiolar stipules connate, foliose bracts obovate to widely elliptic, petiole sericeous (T) and lacks outgrowths between dorsal and lateral wings of samaroid mericarps. It resembles T. discolor, which also shows interpetiolar stipules connate and foliose bracts obovate to widely elliptic. However, in T. discolor the petiole is glabrous and the outgrowths between dorsal and lateral wings of samaroid mericarps are present. In the Midwest it flowers from May to April and fruits in March.

3. Tetrapterys discolor (G. Mey.) DC. in Prodr. 1: 587. 1824. Figs 1 and $3 \mathrm{~A}-\mathrm{D}$

Liana, stem glabrous, smooth or slightly sulcate. Leaves opposite; foliar lamina $7.7-12.4 \mathrm{~cm}$ long, $2.5-4.0 \mathrm{~cm}$ wide, narrow-elliptic to lanceolate, base acute, apex acuminate, margin plane, with glands between the primary vein and margin abaxially, glabrous on both sides; petiole 5.0-8.2 $\mathrm{mm}$ long, glabrous, eglandular; stipules approximately 2.0 $\mathrm{mm}$ long, interpetiolar, connate, triangular, deciduous, older nodes retain a scar. Inflorescence racemose ending on umbels; foliose bracts $5.0-15.0 \mathrm{~mm}$ long, $3.0-12.0 \mathrm{~mm}$ wide, obovate to widely elliptic; bracts $1.2-1.5 \mathrm{~mm}$ long, lanceolate; peduncle 3.0-5.0 mm long, glabrous to glabrescent $(\mathrm{T})$; bracteoles $1.0-1.4 \mathrm{~mm}$ long, eglandular; pedicel $3.0-5.0 \mathrm{~mm}$ long, glabrous to glabrescent (T). Sepals $2.0-2.5 \mathrm{~mm}$ long, $1.5-2.2 \mathrm{~mm}$ wide, ovate, sericeous ( $\mathrm{T}$ and $\mathrm{V}$ ), 8-glandular, glands 1.5-2.4 $\mathrm{mm}$ long, $0.8-1.0 \mathrm{~mm}$ wide, adpressed, elongate. Floral buds yellow. Petals slightly indented at margin, lateral petals, limb $3.0-3.5 \mathrm{~mm}$ long, $2.0-2.8 \mathrm{~mm}$ wide, claw $1.2-2.0 \mathrm{~mm}$ long, posterior petal, limb $3.0-3.2 \mathrm{~mm}$ long, $2.0-3.0 \mathrm{~mm}$ wide, claw $1.8-2.0 \mathrm{~mm}$ long. Stamens heteromorphic; filaments 1.6-2.0 mm long; anthers 1.0-1.2 mm long. Ovary 1.3-1.5 mm long, tomentose ( $\mathrm{T}$ and $\mathrm{V}$ ); styles $1.8-2.0 \mathrm{~mm}$ long, subequal. Samaroid mericarp with four lateral wings, differing from the dorsal wing, glabrous, superior lateral wings 12.0-17.0 $\mathrm{mm}$ long, $6.0-8.0 \mathrm{~mm}$ wide, inferior lateral wings $7.0-10.0$ $\mathrm{mm}$ long, $4.5-5.0 \mathrm{~mm}$ wide, dorsal wing $1.9-2.0 \mathrm{~mm}$ long, $7.0-11.0 \mathrm{~mm}$ wide, with outgrowths between dorsal and lateral wings, nut floccose (T).

Specimens examined: BRAZIL. Mato Grosso: Cuiabá, 14/VI/1902, fr., G.O. Malme 1739 (R); 16/V/1979, fl., M. Macedo et al. 1134 (UFMT); Livramento, 23/VI/1995, fr., M. Macedo \& R. Godinho 4246 (UFMT).

Additional specimens examined: BRAZIL. Maranhão: Carolina, 06/VII/1993, fr., J.A. Ratter et al. 6771 (UB). Tocantins: Araguatins, 27/IV/1961, fr., E. Oliveim 1596 (UB).

Tetrapterys discolor occurs at the forest edges of Bolivia, State of Mato Grosso, extending from the Brazilian Amazon to Guatemala and the West Indies and shows petiole glabrous, foliose bracts and presence of outgrowths between dorsal and lateral wings. It resembles T. crispa, but it shows a sericeous petiole and the outgrowths between the dorsal and lateral wings are absent. In the Midwest it flowers on May and fruits from April to July.

4. Tetrapterys hassleriana Nied. in Bull. Herb. Boiss. 2. Ser. 7: 285. 1907. Figs. 1 and 4D

Figs. 1 and $4 \mathrm{D}$

Scandent subshrub, stem glabrous to glabrescent on older branches and sericeous $(\mathrm{T})$ on younger branches, lenticels sometimes present. Leaves opposite; foliar lamina $1.8-4.3 \mathrm{~cm}$ long, $0.5-1.5 \mathrm{~cm}$ wide, elliptic to obovate, base acute, apex acute to acuminate, margin revolute, with glands on the margin, mostly near base, abaxial surface glabrescent (T), adaxial face glabrous; petiole $1.3-2.5 \mathrm{~mm}$ long, sericeous (T), eglandular, stipules $0.2-0.5 \mathrm{~mm}$ long, intrapetiolar, triangular. Inflorescence a simples raceme; bracts approximately $0.5 \mathrm{~mm}$ long, triangular; peduncle 2.0-9.0 mm long, sericeous (T); bracteoles $1.5-5.0 \mathrm{~mm}$ long, glandular; pedicels $5.0-9.0 \mathrm{~mm}$ long, sericeous (T). Sepals ovate, sericeous (T), 8-glandular, adpressed, elongated. Floral buds yellow. Petals slightly indented at margin. Stamens heteromorphic; filaments approximately $2.0 \mathrm{~mm}$ long; anthers approximately $0.8 \mathrm{~mm}$ long. Ovary tomentose (V); styles approximately $3.0 \mathrm{~mm}$ long, alike or subequal. Samaroid mericarps with four lateral wings, differing from the dorsal wing, glabrescent $(\mathrm{T})$, superior lateral wings 7.5-8.0 mm long, $1.7-2.2 \mathrm{~mm}$ wide, inferior lateral wings 7.5-9.0 mm long, $1.9-2.8 \mathrm{~mm}$ wide, dorsal wing $2.4-3.0 \mathrm{~mm}$ long, 5.4-6.5 mm wide; nut glabrescent to pubescent (T).

Specimens examined: BRAZIL. Mato Grosso: Santa Terezinha, 10/XI/1985, fl., J. Pirani 1176 (INPA). Mato Grosso do Sul: Corumbá, 23/II/1996, fl. fr., A. Pott 7654 (SP); 15/XII/1986, fl., C.N. da Cunha 2169 (SP). Miranda, 13/V/1976, fl., G. Hatschbach 38636 (MBM). 
Tetrapterys hassleriana occurs on flooded lowland in the Pantanal region of Brazil and Paraguay, and in the Araguaia River (Mato Grosso). This species resembles T. microphylla an erect shrub, abaxial surface of lamina sericeous and restricted to highland cerrados and campos rupestres on Chapada dos Veadeiros (Goiás). T. hassleriana is a scandent shrub, abaxial surface of lamina glabrescent occurring in the Pantanal (Mato Grosso do Sul). In the Midwest it flowers from May to December and fruits in February.

5. Tetrapterys jussieuana Nied. in Das Pflanzenreich 141: 169. 1928.

Figs. 1 and $4 \mathrm{C}$

Subshrub, $0.2-0.5 \mathrm{~m}$, stem sericeous (T). Leaves opposite, rarely subopposite. Foliar lamina $2.1-8.0 \mathrm{~cm}$ long, $0.5-2.5 \mathrm{~cm}$ wide, elliptic to obovate, base acute, apex acute to slightly cuspidate, margin plane to slightly revolute with glands on the margin at base, abaxial surface sericeous $(\mathrm{T})$, adaxial surface slightly scabrous $(\mathrm{T})$, hairs short, base up to $0.1 \mathrm{~mm}$ long and branches up to $1.2 \mathrm{~mm}$ long; petiole $2.5-3.0 \mathrm{~mm}$ long, sericeous $(\mathrm{T})$, glands rarely at apex; stipules $0.7-1.2 \mathrm{~mm}$ long; intrapetiolar, triangular. Inflorescence a simple raceme, lax; bracts $0.8-1.6 \mathrm{~mm}$ long, elliptic; peduncle $2.5-3.5 \mathrm{~mm}$ long, sericeous $(\mathrm{T})$; bracteoles $1.2-2.3 \mathrm{~mm}$ long, glandular at base; pedicels $2.1-4.0 \mathrm{~mm}$ long, sericeous (T). Sepals $2.5-3.0 \mathrm{~mm}$ long, $1.3-2.2 \mathrm{~mm}$ wide, ovate, sericeous (T), 8-glandular, $2.0-2.3 \mathrm{~mm}$ long, 1.1-1.5 mm wide, adpressed, elongate. Flower buds orange to yellow. Petals slightly indented at margin, sometimes orange at primary veins, lateral petals, limb $4.5-5.6 \mathrm{~mm}$ long, 3.2-4.5 mm wide, claw $1.5-2.5 \mathrm{~mm}$ long, posterior petal, limb 5.0-6.0 mm long, 3.0-4.0 mm wide, claw 1.5-2.7 $\mathrm{mm}$ long. Stamens heteromorphic; filaments $1.3-2.5 \mathrm{~mm}$ long, anthers $1.0-1.3 \mathrm{~mm}$ long. Ovary $1.0-1.2 \mathrm{~mm}$ long, tomentose ( $\mathrm{T}$ and $\mathrm{V}$ ), with lateral wings; styles $2.2-3.6 \mathrm{~mm}$ long, alike. Samaroid mericarps with two lateral wings, alike the dorsal wing, sericeous (T), lateral wings $6.0-11.0 \mathrm{~mm}$ long, $14.0-23.0 \mathrm{~mm}$ wide, dorsal wing $6.0-11.0 \mathrm{~mm}$ long, 11.0-20.0 mm wide; nut sericeous (T).

Specimens examined: BRAZIL. Distrito Federal: Brasília, 18/X/2003, fl. fr., F.F.O. Pereia et al. 2 (UB); 25/IX/2003, fl., G.A. Pereira et al. 18 (UB); 3/XI/2005, fl., G.H. Rua 662 (CEN); 9/VI/1999, fl., J.G. Faria \& J.B. Ferreira 177 (CEN); 16/X/2007, fr., P.S. Carvalho \& C.A.S Correia 345 (UB); 16/X/2007, fr., P.S. Carvalho \& C.A.S Correia 346 (UB); 1/XI/2002, fr., R. Rodrigues-da-Silva et al. 736 (HEPH). Brazlândia, 28/IX/2004, fl., A.H. Salles et al. 3143 (HEPH). Taguatinga, 14/X/1996, fl., S.M. Gomes \& R.G. Tostes 161 (CEN). Goiás: Alto Paraíso de Goiás, 15/X/2010, fl., A. Francener 1020 (UFG, CGMS); 30/VIII/1998, fr., K.A.C. Rosa et al. 11 (HEPH). Caiapônia, 17/XI/2007, fr., S.S. Silva et al. 484 (IBGE). Caldas Novas, 22/IX/1993, fr., G.P. da Silva \& O. Fumagali 1936 (CEN). Cavalcante, 13/X/2000, fl., F. Bucci 1463 (UFG). Chapada dos Veadeiros, 7/X/1972, fr.,
J. A. Rizzo 8451 (UFG); 20/VII/1994, fl. fr., M. Aparecida da Silva et al. 2174 (IBGE). Cristalina, 15/VIII/1980, fl., G. Hatschbach 43129 (MBM). Leopoldo Bulhões, 28/IX/1993, fr., J.T. Chaves-Filho et al. 5 (UFG); Luziânia, 23/X/ 2007, fl., C.H.G. Cezare \& A. Machado-Neto 287 (UB). Minaçu, 9/X/1991, fr., T.B. Cavalcanti et al. 876 (CEN). Niquelândia, 17/IX/1998, fl., E.L. Jacques et al. 800 (CEN, SP); 6/X/1995, fr., T.B. Cavalcanti et al. 1794 (CEN). Região do Maranhão Superior, 01/IX/1892, fr., E. Ule s/n (R). Pirenópolis, 4/ VIII/1971, fl., J.A. Rizzo \& A. Barbosa 6604 e 5853 (UFG); 18/IX/2010, fr., R.D. Sartin 89 (UFG); 18/IX/2010, fl. fr., R.D. Sartin 90 et al. (UFG); 9/X/2010, fr., R.D. Sartin, 112 (UFG); 9/X/2010, fr., R.D. Sartin 113 (UFG); 09/X/2010, fr., R.D. Sartin 114 (UFG). São João d'Aliança, 12/X/2006, fl., H.D. Ferreira 4509 (UFG). Mato Grosso do Sul: Campo Grande, 4/IX/1936, fl., W.A. Archer \& A. Gehrt s/n (SP).

Tetrapterys jussieuana is restricted to the Brazilian Cerrado (cerrado sensu strictu, campo rupestre and cerrado rupestre), from Parana to both Tocantins and Bahia States, usually after fires. Its foliar lamina is sericeous $(\mathrm{T})$, resembling T. microphylla, but differing by being a subshrub up to $0.6 \mathrm{~m}$ tall (from 0.7 to $2.0 \mathrm{~m}$ tall in T. microphylla), and samaroid mericarps with two lateral wings alike the dorsal wing (four lateral wings in T. microphylla). It is most easily misidentified as T. ambigua, but differs from it by the abaxial surface of laminas sericeous ( $\mathrm{T}$ ), and adaxially scabrous (T) (villous to lanose (T) in T. ambigua), hairs with base and branches short (base up to $0.1 \mathrm{~mm}$ long, and branches $1.2 \mathrm{~mm}$ long) (hairs with base and branches long, up to $0.2 \mathrm{~mm}$ long at base, and $2.0 \mathrm{~mm}$ long at branches). In the Midwest, it flowers from July to November and fruits from July to December.

6. Tetrapterys microphylla (A.Juss.) Nied. in Verz. Vorles. Lyc. W.-S. 1909/10: 5. 1909.

Figs. 1 and $5 \mathrm{~A}-\mathrm{G}$

Shrubs $0.7-2.2 \mathrm{~m}$ tall, stem glabrous to glabrescent (T), presenting longitudinal clefts, mostly on distal nodes, away from the inflorescence. Leaves opposite; foliar lamina $1.5-3.9 \mathrm{~cm}$ long, $0.3-1.3 \mathrm{~cm}$ wide, narrow-elliptic, elliptic to obovate, base acute, apex acuminate to apiculate, margin revolute, glandular at margin abaxially, mostly near base, abaxial surface sericeous (T), adaxial surface glabrescent to sericeous (T); petiole $2.0-3.0 \mathrm{~mm}$ long, sericeous $(\mathrm{T})$, eglandular; stipules $0.1-0.2 \mathrm{~mm}$ long, intrapetiolar, triangular. Inflorescence a simple raceme, slightly congested; bracts 3.0-3.5 mm long, lanceolate; peduncles $4.0-10.0 \mathrm{~mm}$ long, sericeous ( $\mathrm{T})$; bracteoles $1.5-4.0 \mathrm{~mm}$ long, glandular; pedicels $3.7-7.0 \mathrm{~mm}$ long, sericeous (T). Sepals $3.1-3.5 \mathrm{~mm}$ long, 1.2-2.0 mm wide, ovate, sericeous ( $\mathrm{T})$, 8-glandular, 2.0-2.5 mm long, $0.8-1.0 \mathrm{~mm}$ wide, adpressed, elongate. Flower buds yellow. Petals slightly indented at margin; lateral petals with limb $4.5-5.0 \mathrm{~mm}$ long, 3.1-3.9 $\mathrm{mm}$ wide, claw $1.0-1.3 \mathrm{~mm}$ long, posterior petal with limb 5.0-6.0 


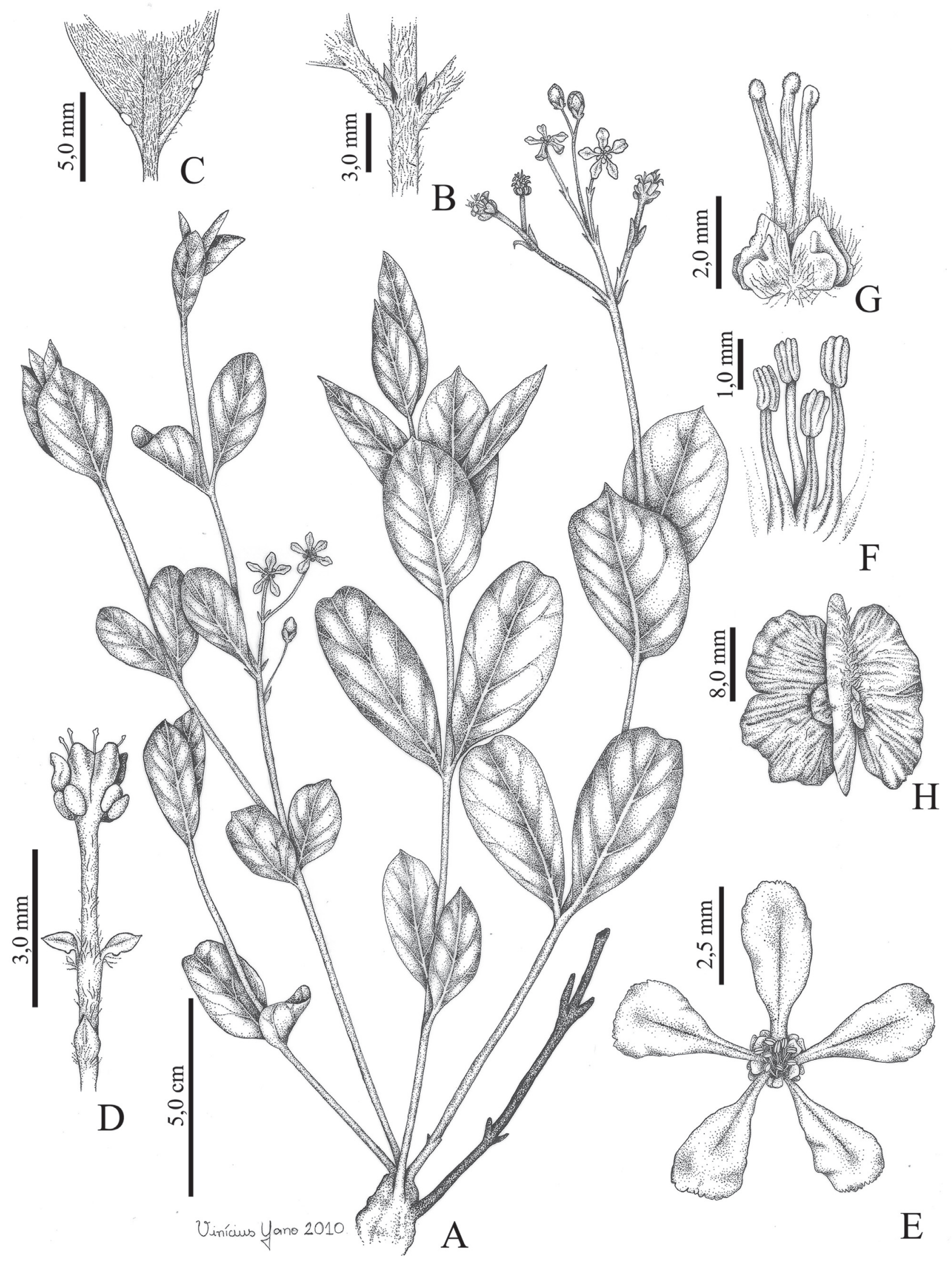

Figure 2. Tetrapterys ambigua A) Habit; B) Intrapetiolar stipules; C) Glands on the margin of foliar lamina; D) Bracts and bracteoles; E) Flower in frontal view; F) Stamens connate at base; G) Ovary with lateral wings; H) Samaroid mericarp in frontal view with four lateral wings, alike dorsal wing (A. Francener et al. 1023). 
mm long, 3.0-3.8 $\mathrm{mm}$ wide, claw $1.5-2.0 \mathrm{~mm}$ long. Stamens heteromorphic; filaments $1.8-2.7 \mathrm{~mm}$ long; anthers 1.0-1.2 $\mathrm{mm}$ long. Ovary $0.9-1.4 \mathrm{~mm}$ long, tomentose $(\mathrm{T})$, lateral wings; styles $2.0-3.3 \mathrm{~mm}$ long, anterior style the longest. Samaroid mericarps with four lateral wings, differing from the dorsal wing, glabrescent $(\mathrm{T})$, superior lateral wings 4.0-5.0 $\mathrm{mm}$ long, $2.5-3.5 \mathrm{~mm}$ wide, inferior lateral wings $4.4-8.5$ $\mathrm{mm}$ long, $3.5-5.0 \mathrm{~mm}$ wide, dorsal wing $1.5-3.0 \mathrm{~mm}$ long, $3.5-4.2 \mathrm{~mm}$ wide, rarely with outgrowths between dorsal and lateral wings; nut tomentose (T).

Specimens examined: BRAZIL. Goiás: Alto Paraíso de Goiás: A. Francener et al. 1028, 16/X/2010, fl. fr., (CGMS, UFG); 26/V/1994, fl., C. Munhoz et al. 162 (UB); 31/V/1997, fl., C. Munhoz et al. 409 (HEPH, UB); 10/VIII/2007, fl. fr., C. Proença \& S.A Harris 3401 (UB); 10/X/1979, fl., E.P. Heringer et al. 2329 (IBGE); 30/XI/1998, fl. fr., R. Kral et al. 75763 (SP); 23/X/1996, fl. fr., R. Marquete et al. 2765 (IBGE); 16/VIII/1995, fl., R. Marquete et al. 2293-A (IBGE); 10/IX/1996, fl., R.C. Mendonça et al. 2626 (IBGE, UFG); 23/VIII/1993, fl., S. Splett 61 (UB); 3/VI/1999, fl. fr., T.B. Cavalcanti \& R.M. Silva 2504 (CEN); 24/VI/1994, fl., V.L. Gomes-Klein et al. 2447 (UFG). Chapada dos Veadeiros, 18/ VII/1964, fl., G.T. Prance \& N.T. Silva s/n (UB); 21/X/1965, fl. fr., H.S. Irwin et al. 9389 (UB); 19/II/1969, fl., H.S. Irwin et al. 23538 (UB); 25/III/1971, fl. fr., H.S. Irwin et al. 33164 (UB); 8/II/1987, fl., J.R. Pirani et al. 1887 (SP); 21/XII/1968, fr., M. Graziela \& A. José s/n (UB); 6/III/1973, fl., W. R. Anderson 6434 (UB); 15/VIII/1990, fl., T. B. Cavalcanti et al. 676 (CEN, HEPH, HUEFS, IBGE).

Tetrapterys microphylla is restricted to Brazil in highland cerrados and campos rupestres in Bahia, Goiás, Minas Gerais and Tocantins. In the Midwest, it is a shrub endemic to Chapada dos Veadeiros. It resembles T. jussieuana, but differs in its habit (a subshrub in T. jussieuana), and on the samaroid mericarps with four lateral wings differing from the dorsal wing (samaroid mericarps with just two lateral wings, alike the dorsal wing in T. jussieuana). In the Midwest it flowers and fruits from June to March.

7. Tetrapterys mucronata Cav. in Monadelphiae Classis Dissertationes Decem 9: 434, tab. 262: 2. 1790.

Figs. 1 and 6A-F

Liana, stem glabrous, sericeous to farinaceous ( $\mathrm{T})$, with small longitudinal clefts. Leaves opposite; foliar laminas 5.9-11.0 cm long, $2.4-7.0 \mathrm{~cm}$ wide, elliptic, base acute, apex cuspidate, margin revolute, glands between primary vein and margin, mostly near base, abaxial surface, glabrous, adaxial surface glabrous to glabrescent $(\mathrm{T})$; petiole 8.0-15.0 mm long, glabrous to glabrescent, eglandular; stipules $0.4-0.6 \mathrm{~mm}$ long, interpetiolar, free, oblong. Inflorescence a raceme ending in umbels; foliose bracts 2.2-9.0 $\mathrm{mm}$ long, $1.5-2.0 \mathrm{~mm}$ wide, lanceolate, a pair of glands at base; bracts $0.6-1.2 \mathrm{~mm}$, lanceolate; peduncle $2.0-3.0 \mathrm{~mm}$ long, glabrescent to sericeous (T); bracteoles $0.6-1.0 \mathrm{~mm}$ long, eglandular; pedicel $2.0-5.5 \mathrm{~mm}$ long, glabrescent to sericeous (T). Sepals 1.6-2.0 mm long, $1.5-1.8 \mathrm{~mm}$ wide, rounded, sericeous $(\mathrm{T}), 8$-glandular, $2.0-2.2 \mathrm{~mm}$ long, $1.8-2.0 \mathrm{~mm}$ wide, stalked, auriculate to absent. Floral buds yellow. Petals entire at margin, lateral petals, limb 4.0-4.7 mm long, 2.5-3.6 $\mathrm{mm}$ wide, claw 1.4-2.0 $\mathrm{mm}$ long, posterior petal, limb 2.3-3.0 mm long, 2.2-2.5 mm wide, claw 2.1-2.5 $\mathrm{mm}$ long. Stamens near posterior petal smaller than others; filaments 1.0-3.0 mm long; anthers $1.3-1.9 \mathrm{~mm}$ long. Ovary 0.9-2.0 mm long, glabrous, with lateral wings; styles 2.9-5.5 $\mathrm{mm}$ long, unequal. Samaroid mericarps with four lateral wings, differing from the dorsal wing, glabrous, superior lateral wings $6.0-12.6 \mathrm{~mm}$ long, $1.7-3.3 \mathrm{~mm}$ wide, inferior lateral wings $4.2-5.6 \mathrm{~mm}$ long, $1.5-2.1 \mathrm{~mm}$ wide, dorsal wing 1.6-5.0 $\mathrm{mm}$ long, $4.0-5.0 \mathrm{~mm}$ wide, with outgrowths between the dorsal wing and lateral wings; nut glabrous.

Specimens examined: BRAZIL. Distrito Federal: unkown locality, 17/X/1979, fl., O.A. Costa s/n (R). Mato Grosso: Cáceres, 1/VII/1911, fl., Comissão Rondon 4084 (R); 11/VIII/2002, fr., M. Schessl 6154 (HPAN). Mundo Novo, 27/VIII/2008, fr., D. Sasaki 2461 (HERBAM); 12/ II/2009, fr., D. Zappi 1236 (HERBAM) São Luis de Cáceres, 21/VIII/1908, fl. fr., F.C. Hoehne 180 (R); São Luis de Cáceres, 21/VIII/1908, fl. fr., F.C. Hoehne 181 (R).

Additional specimens examined: BRAZIL. Amazonas: Reserva Florestal Ducke, 5/VI/1993, fl., J.E.L.S Ribeiro et al. 875 (UFMT). Bahia: Umburanas, 13/VIII/1999, fl., E. de Melo et al. 2790 (HUEFS); 13/VIII/1999, fl., E. de Melo et al. 2828 (HUEFS). Espírito Santo: Conceição do Castelo, 20/VIII/1987, fl. fr., G. Hatschbach et al. 51316 (UB). Minas Gerais: Marliéria, 19/IX/1975, fl. fr., E.P. Heringer \& G. Eiten 15067 (UB). Rio de Janeiro: São Gonçalo a Laranjal, 26/VII/1876, fl., A.F.M. Glaziou 8585 (R).

Tetrapterys mucronata Cav. occurs in the forest in the entire Brazil, and in Bolivia extending to Panama and the lesser Antilles. It resembles T. crispa and T. discolor by the foliose bracts. However, T. mucronata differs from them by showing interpetiolar stipules free, and sepals with adpressed, auriculate glands (interpetiolar stipules connate and sepals with adpressed and elongate glands in T. crispa and T. discolor). In the Midwest it flowers from October to June and fruits from August to September.

8. Tetrapterys racemulosa A.Juss. in. Arch. Mus. III. 547. 1843.

Shrub 0.5-0.7 m, stem tomentose (T). Leaves opposite; foliar lamina $3.0-6.3 \mathrm{~cm}$ long, $1.2-3.0 \mathrm{~cm}$ wide, elliptic, base cuneate, apex acuminate, margin plane or slightly revolute, glandular at margin, mostly near base, abaxial surface tomentose $(\mathrm{T})$, adaxial surface pubescent $(\mathrm{T})$, mostly near veins; petiole 4.0-7.0 mm long, tomentose (T), eglandular; stipules $1.2-1.5 \mathrm{~mm}$ long, intrapetiolar, lanceolate. Inflorescence a simple raceme, lax; bracts 4.0-5.0 $\mathrm{mm}$ long, lanceolate; peduncles $3.0-7.0 \mathrm{~mm}$ long, velutinous to tomentose (T); bracteoles $2.0-3.0 \mathrm{~mm}$ long, glands at base or middle 


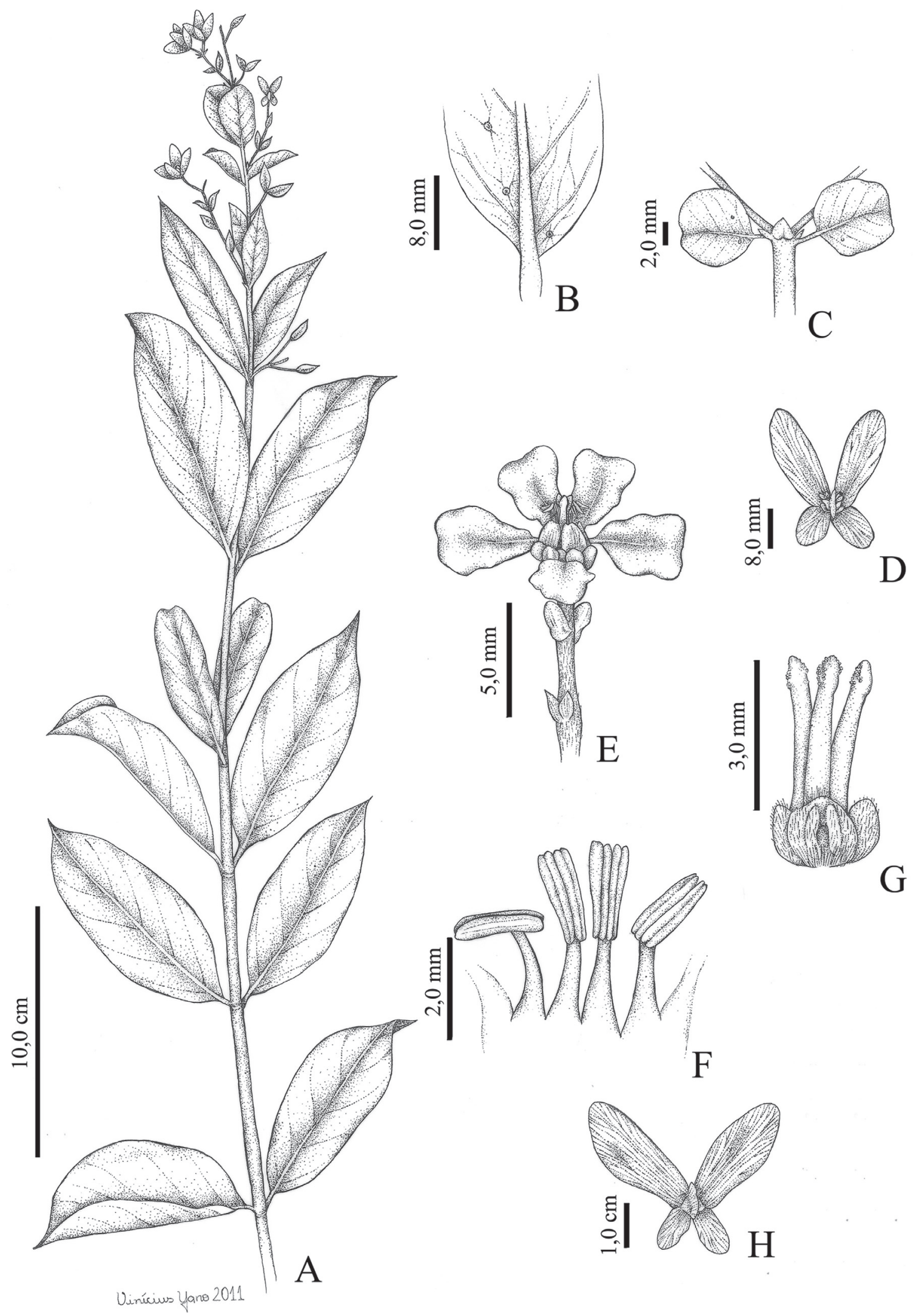

Figure 3. Tetrapterys discolor A) Habit; B) Glands on foliar lamina; C) Interpetiolar stipules connate; D) Samaroid mericarp in frontal view with outgrowths between dorsal and lateral wings (M. Macedo \& R. Godinho 4246). T. crispa E) Flower with bracts and bracteoles; F) Stamens connate at base; G) Ovary with lateral wings; H) Samaroid mericarps in frontal view without outgrowths between dorsal and lateral wings (B. Dubs 2159). 


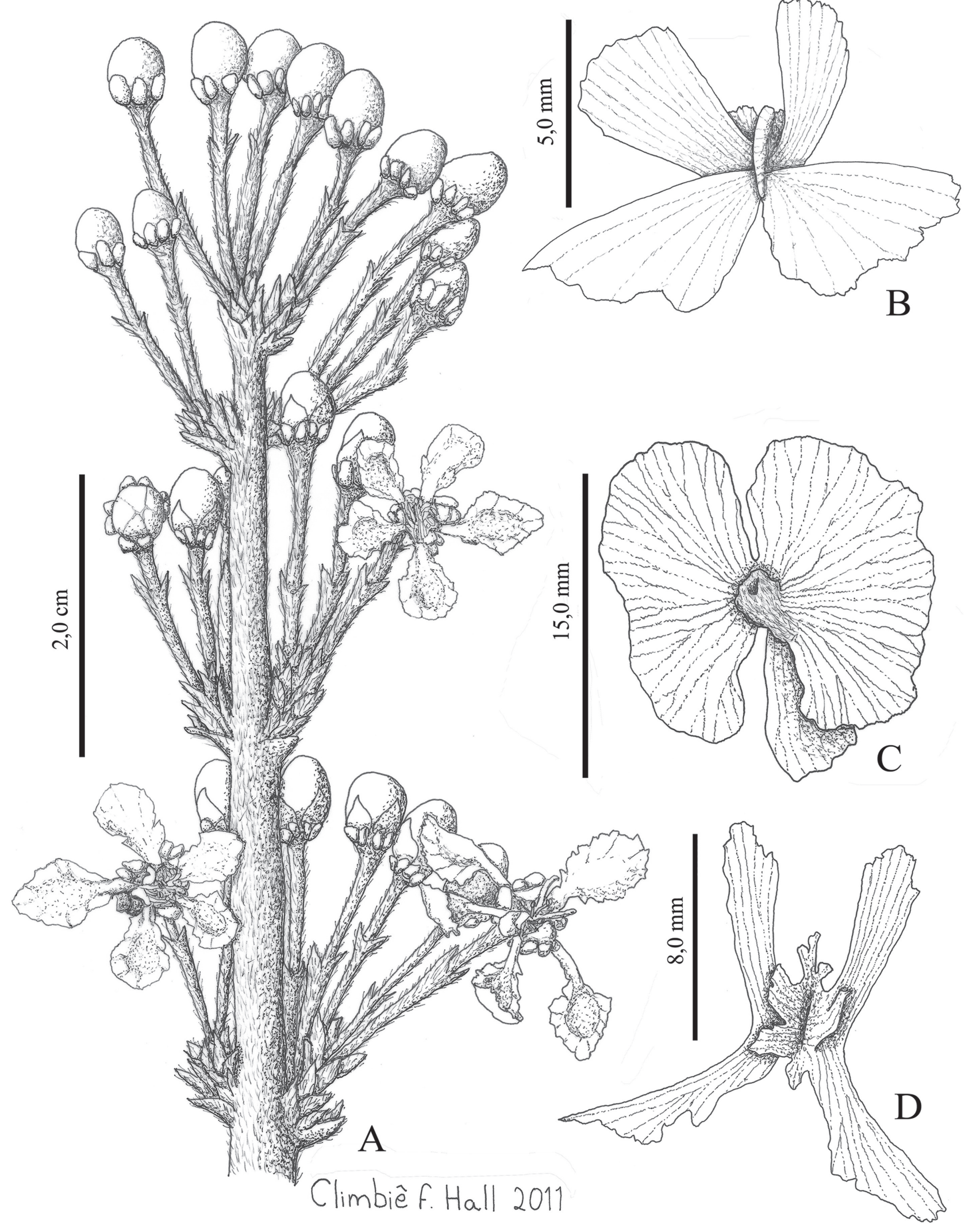

Figure 4. Tetrapterys ramiflora A) Habit (M.C. Andrade s/n); B) Samaroid mericarp in frontal view with lateral wings differing from the dorsal wing (R.D. Sartin 103); T. jussieuana C), Samaroid mericarp in frontal view with four lateral wings, alike the dorsal wing. (A. Francener et al. 1020). T. hassleriana D) Samaroid mericarp in frontal view with lateral wings differing from the dorsal wing (A. Pott 7654). 


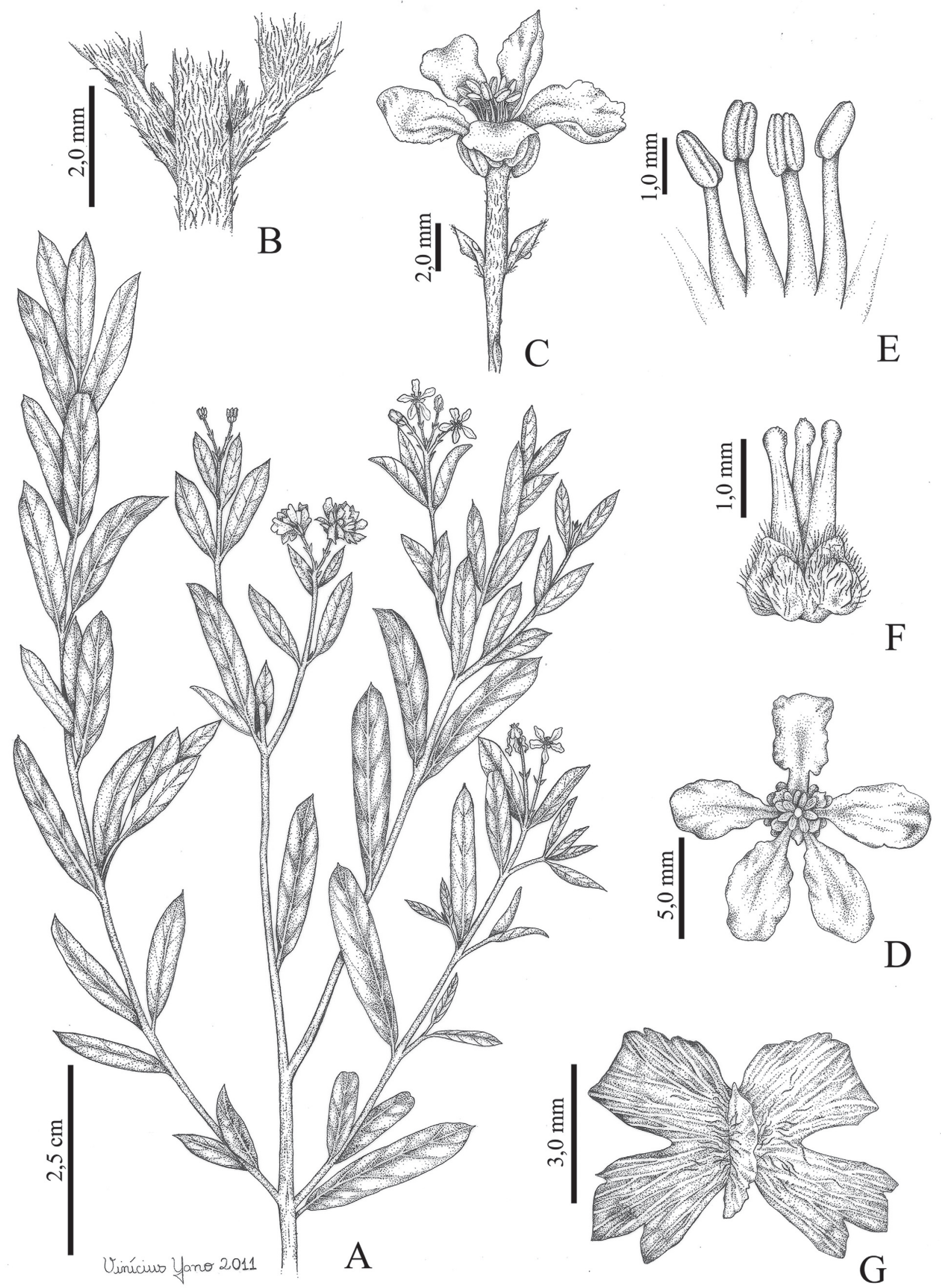

Figure 5. Tetrapterys microphylla A) Habit; B) Interpetiolar stipules; C) Flower with bracts and bracteoles; D) Flower in frontal view; E) Stamens connate at base; F) Ovary with lateral wings; G) Samaroid mericarp in frontal view with lateral wings differing from the dorsal wing (A. Francener et al. 1028). 

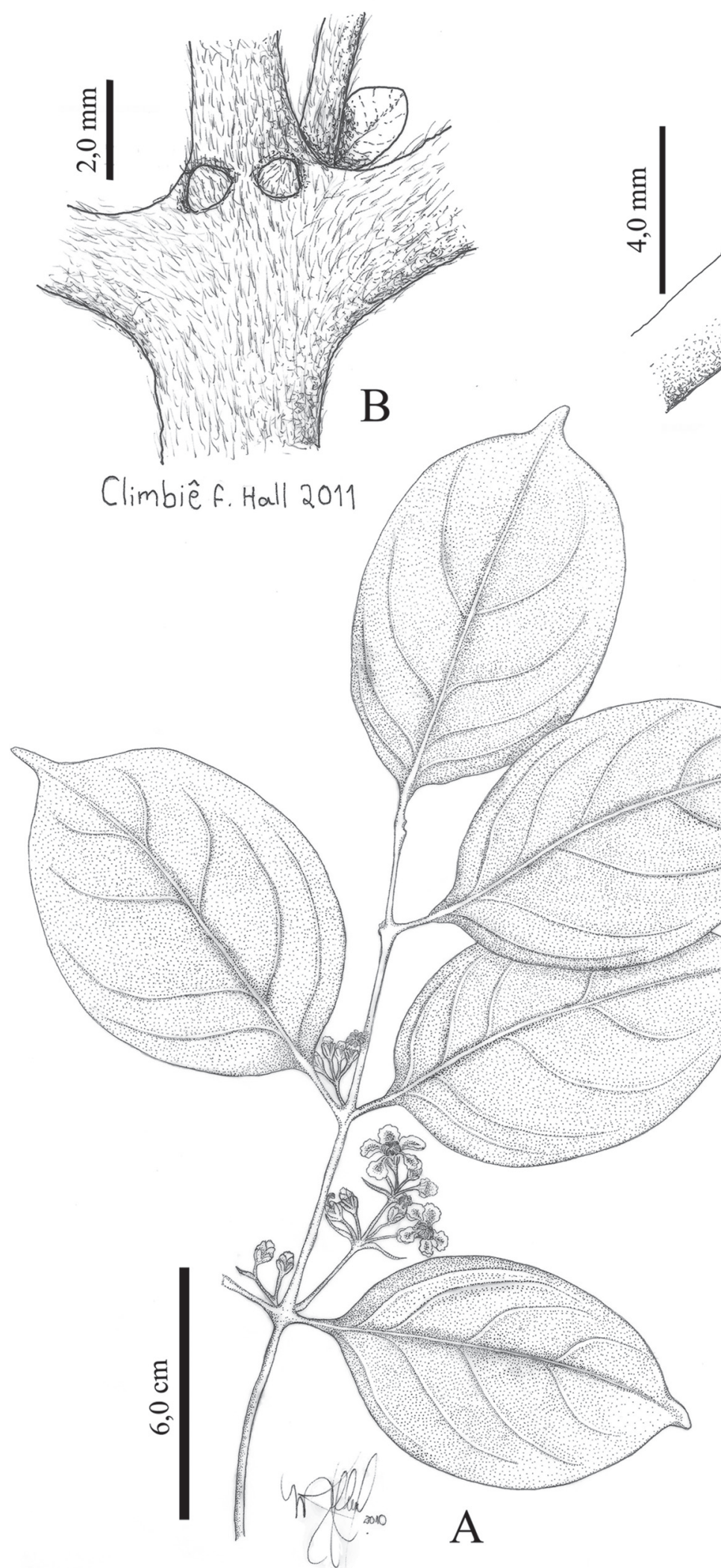

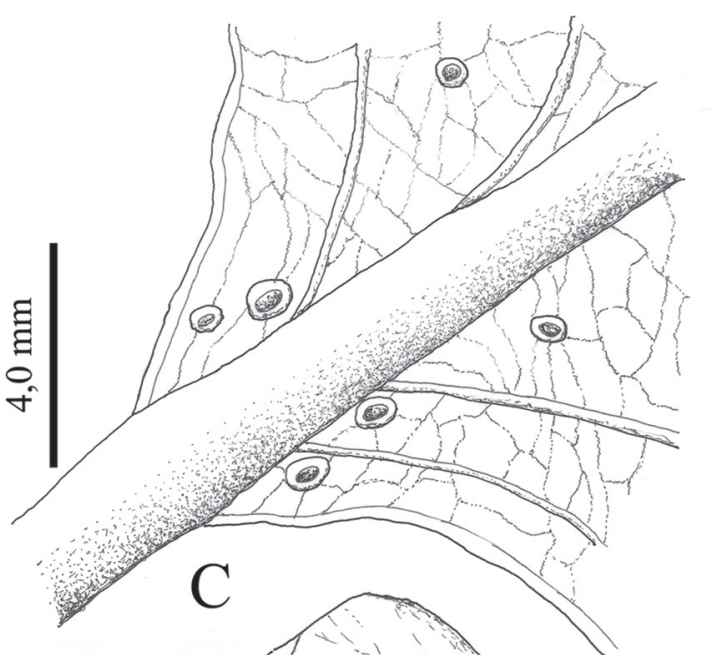


when present; pedicels $7.0-10.0 \mathrm{~mm}$ long, velutinous to tomentose (T). Sepals 3.2-3.9 mm long, $1.4-1.5 \mathrm{~mm}$ wide, triangular, sericeous (T), 8-glandular, glands $1.8-2.2 \mathrm{~mm}$ long, 0.9-1.0 mm wide, adpressed, elongate. Flower buds yellow. Petals slightly indented at margin, lateral petals, limb 4.0-4.4 mm long, $3.0-3.7 \mathrm{~mm}$ wide, claw $0.5-1.2 \mathrm{~mm}$ long, posterior petal, limb $4.0-4.2 \mathrm{~mm}$ long, $2.9-3.0 \mathrm{~mm}$ wide, claw 1.4-1.5 mm long. Stamens heteromorphic; filaments 1.1-1.8 mm long; anthers $1.5-1.7 \mathrm{~mm}$ long. Ovary $1.0-1.4$ $\mathrm{mm}$ long, velutinous $(\mathrm{T})$, with lateral wings; styles $2.0-2.2$ $\mathrm{mm}$ long, alike. Samaroid mericarps with four lateral wings, alike the dorsal wing, glabrescent to pilose $(\mathrm{T})$, superior lateral wings $6.0-11.0 \mathrm{~mm}$ long, $6.0-9.0 \mathrm{~mm}$ wide, inferior lateral wings $7.0-10.0 \mathrm{~mm}$ long, $6.0-10.0 \mathrm{~mm}$ wide, dorsal wing $11.0-13.0 \mathrm{~mm}$ long, $6.7-9.0 \mathrm{~mm}$ wide, with lateral outgrowths; nut tomentose (T).

Specimens examined: BRAZIL. Mato Grosso do Sul: Corumbá. 31/X/2010, fl. fr., F.G.M. Caporal 1007 (CGMS).

Additional specimens examined: BOLÍVIA. Provincia de Nuflo de Chávez: San Antonio. 12/XII/1993, fr., M. Toledo \& G. Chuvé 333 (USZ).

Tetrapterys racemulosa occurs in wooded savanna and in Cerrado sensu strictu of Brazil and Bolivia. In Brazil is is only present in the State of Mato Grosso do Sul. It shows a lax racemose inflorescence, resembling T. ambigua and T. jussieuana, with all of them presenting samaroid mericarps with lateral wings alike the dorsal wing. However, $T$. racemulosa shows a samaroid mericarp with four lateral wings (two superior and two inferior wings), while T. ambigua and T. jussieuana show a samaroid mericarp with just two lateral wings. In the Midwest it flowers and fruits in October.

9. Tetrapterys ramiflora A.Juss. in. A. St-Hil. Fl. Bras. Mer. 3: 8.1832.

Figs. 1 and $4 \mathrm{~A}-\mathrm{B}$

Subshrub to shrub 1.0-2.2 m tall, stem glabrescent to tomentose (T), sometimes with scattered lenticels. Leaves opposite; foliar lamina $5.0-12.0 \mathrm{~cm}$ long, $1.5-5.3 \mathrm{~cm}$ wide, elliptic, base acute, apex acuminate, margin plane to revolute, with a par of glands at margin, mostly near base, abaxial surface glabrescent to tomentose (T), hairs most densely arranged near primary veins, adaxial surface tomentose (T); petiole 4.5-10.0 mm long, tomentose $(\mathrm{T})$, eglandular or 1-2-glandular; stipules $1.3-4.0 \mathrm{~mm}$ long, intrapetiolar, triangular. Inflorescence a raceme of cincinii, sessile, congested at stem (cauliflorous); bracts $2.0-3.8 \mathrm{~mm}$, triangular, sometimes with many glands; peduncle $2.5-10.8 \mathrm{~mm}$ long, tomentose ( $\mathrm{V}$ and $\mathrm{Y}$ ); bracteoles $2.3-3.3 \mathrm{~mm}$ long, glands usually at base or absent; pedicel $3.5-12.0 \mathrm{~mm}$ long, tomentose ( $\mathrm{V}$ and $\mathrm{Y}$ ). Sepals $2.8-4.5 \mathrm{~mm}$ long, $1.0-2.2 \mathrm{~mm}$ wide, ovate, sericeous (T), 8-glandular, glands 1.7-2.9 mm long, $0.8-1.3 \mathrm{~mm}$ wide, adpressed, elongate. Floral buds orange to yellow. Petals slightly indented at margin, sometimes with orange veins; lateral petals, limb $3.7-7.7 \mathrm{~mm}$ long, 3.3-4.6 mm wide, claw $0.8-1.7 \mathrm{~mm}$ long, posterior petal, limb 4.0-6.0 mm long, 3.0-4.0 mm wide, claw $1.5-2.5 \mathrm{~mm}$ long. Stamens heteromorphic; filaments $0.8-3.3 \mathrm{~mm}$ long; anthers $0.8-1.3 \mathrm{~mm}$ long. Ovary $1.2-1.8 \mathrm{~mm}$ long, tomentose ( $\mathrm{T}$ and $\mathrm{V}$ ), with lateral wings; styles $2.2-3.0 \mathrm{~mm}$ long, equal to subequal. Samaroid mericarps with four lateral wings, differing from the dorsal wing, glabrous, glabrescent to tomentose $(\mathrm{V})$, superior lateral wings $3.2-8.6 \mathrm{~mm}$ long, 1.4-6.0 mm wide, inferior lateral wings $2.5-10.0 \mathrm{~mm}$ long, 2.7-6.0 mm wide, dorsal wing 1.5-4.0 mm long, 2.1-6.5 $\mathrm{mm}$ wide, sometimes with outgrowths between the dorsal and lateral wings; nut glabrescent (V and $\mathrm{Y}$ ).

Specimens examined: BRAZIL: Distrito Federal: Brasília, 15/X/2003, fr., C. Proença et al. 2704 (UB); 26/ VII/1990, fl. fr., L.B. Bianchetti et al. 912 (CEN); 13/IX/2008, fl. fr., M. Aparecida da Silva 6587 (IBGE); 17/VIII/2000, fl., M.G. Nobrega 1270 (HEPH); Gama, 17/IX/1996, fl. fr., S.M. Gomes et al. 72 (CEN). Goiás: Aparecida de Goiânia, 30/VI/2003, fl. fr., J.B.A. Bringel \& J.F.B. Pastore 34 (CEN). Caldas Novas, 16/VIII/2008, fl. fr., A. Francener et al. 630 (UFG). Goiânia, 6/VIII/1968, fl., J. A. Rizzo \& A. Barbosa 1912 (UFG); 27/IX/2010, fl. fr., R.D. Sartin 103 (UFG). Goiânira, 22/VIII/1970, fl., J.A. Rizzo 5436 (UFG). Morrinhos, 28/VIII/1970, fl., J. A. Rizzo 5469 (UFG). Pirenópolis, 22/IX/2008, fl. fr., A. Francener 663 (UFG); 18/IX/2010, fr., R.D. Sartin 92 (UFG). Mato Grosso: Acorizal, 24/VIII/1984, fr., L. Coradin et al. 6876 (CEN). Araguainha, 31/VII/2009, fl. fr., A. Francener et. al. 839 (CGMS, UFG). Barra do Garças, 15/IX/1968, fl., G. Eiten \& L.T. Eiten 8789 (SP). Cáceres, 09/VI/2002, fl. Fr., M. Schessl 6022 (HPAN). Cuiabá, 30/ IX/1980, fl., A.L. Prado et al. s/n (UFMT); 8/IX/1992, fl., G.L. Webster 29729 (UB). 20/IX/1968, fl. fr., R.M. Harley \& R. Souza 10141 (UB). Nova Mutum, 12/VIII/2010, fl. fr., A. Francener \& A.F.N. Gonzaga 996 (CGMS, UFG, UFMT). Mato Grosso do Sul: Alcinópolis, 11/X/2007, fl. fr., A. Pott 14586 (CGMS, UFG). Corguinho, 15/V/2009, fl., M.C. Andrade s/n (CGMS). Sonora, 5/VIII/2001, fl. fr., U.M. Resende \& S. Aragaki 2681 (CGMS). Três Lagoas, 24/V/1993, fl., A.D. Caliente et al. 1152 (SP).

Additional specimens examined: BRAZIL: São Paulo: Botucatu, 22/X/1971, fl., I.S. Gottsberger, 2166 (UB).

Tetrapterys ramiflora is endemic to Brazil and mostly occurs in Cerrado areas. It is a shrubby species that loses its leaves when flowering and fruiting. It differs from all Tetrapterys species in the Midwest by the inflorescence in racemes of cincinii. In the Midwest, it flowers from May to October and fruits from June to October.

\section{Acknowledgments}

The authors thank the curators and staff of the visited herbaria for the loan of specimens, Coordenação de Aperfeiçoamento de Pessoal de Nível Superior (CAPES) for the scholarship granted to the first author; Programa de Pós- 
Graduação em Biologia Vegetal from Universidade Federal do Mato Grosso do Sul (UFMS), Universidade Federal de Goiás (UFG) for logistic support, Climbiê Ferreira Hall, Milena Diniz and Vinícius Yano Corrêa for the drawings, and Dr. Maria Ana Farinaccio for the valuable corrections.

\section{References}

Anderson C. 1997. Revision of Pterandra (Malpighiaceae). Contribution of the University of Michigan Herbarium 21: 1-27.

Anderson WR. 1979. Floral conservatism in neotropical Malpighiaceae. Biotropica 11: 219-223.

Anderson WR. 1981. Malpighiaceae. The Botany of Guayana Highland - Part IX. Memoirs of the New York Botanical Garden 32: 21-305.

Anderson WR. 1998. Malpighiaceae. In: Dubs B. (ed.) Prodromus Florae Matogrossensis. 1 ed. Küsnacht, Betrona-Verlag p. 233-239.

Anderson WR. 2006. Eight segregates from the neotropical genus Mascagnia (Malpighiaceae). Novon 16: 168-204.

Anderson WR, Anderson C, Davis CC. 2006. Malpighiaceae. http://herbarium.Isa.umich.edu/malpigh. 3 Mar. 2010

Bridson D, Forman L. 1992. The Herbarium Handbook. Richmond, Royal Botanic Gardens.

Brummitt RL, Powell CE. 1992. Authors of plant names. London, Royal Botanic Gardens.

Cameron KM, Chase MW, Anderson WR, Hills HG. 2001. Molecular Systematics of Malpighiaceae: Evidence from plastid Rbcl and Matk sequences. American Journal of Botany 88: 1847-1862.

Cavalcanti TB, Ramos AE. 2001. Flora do Distrito Federal, Brasil. Vol. 1. Brasília, Embrapa Recursos Genéticos e Biotecnologia.

Davis CC, Anderson WR. 2010. A complete generic phylogeny of Malpighiaceae inferred from nucleotide sequence data and morphology. American Journal of Botany 97: 2031-2048.

Davis CC, Anderson WR, Donoghue M. 2001. Phylogeny of Malpighi- aceae: Evidence from Chloroplast NDHF AND TRNL-F Nucleotides Sequences. American Journal of Botany 88: 1830-1846.

Gates B. 1982. Banisteriopsis, Diplopterys (Malpighiaceae). Flora Neotropica. Vol. 30, New York, NYBG Press.

IBGE - Instituto Brasileiro de Geografia e Estatística. 2009. http://www. ibge.gov.br/estadosat. 25 Aug. 2009.

Mamede MCH. 1984. Flora Fanerogâmica da Reserva do Parque Estadual das Fontes do Ipiranga (São Paulo, Brasil): 125 - Malpighiaceae. Hoehnea 11: 108-113.

Mamede MCH. 1987. Flora da Serra do Cipó, Minas Gerais: Malpighiaceae. Boletim de Botânica da Universidade de São Paulo 9: 157-198.

Mamede MCH. 1990. Revisão do Gênero Camarea Saint-Hilaire (Malpighiaceae). Hoehnea 17: 1-34.

Mamede MCH. 2014. Tetrapterys in Lista de Espécies da Flora do Brasil. Jardim Botânico do Rio de Janeiro. http://floradobrasil.jbrj.gov. br/2010/FB008967. 4 Apr. 2014.

Mendonça RC, Felfili JM, Walter BMT, et al. 2008. Flora vascular do Bioma Cerrado: In: Sano SM, Almeida SP, Ribeiro JF. (eds.). Cerrado: ecologia e flora. Vol. 2. Brasília, Embrapa Informação Tecnológica. 876 p.

Niedenzu F. 1928. Malpighiaceae. In: Engler A. (ed.) Das Pflanzenreich. Wilhelm Engelmann. Leipzig v. 141, 870 p.

Radford AE, Dickison WC, Massey JR, Bell CR. 1974. Vascular Plant Systematics. New York, Harper \& Row Publishers.

SpeciesLink. 2010. http://splink.cria.org.br. 10 Nov. 2009.

Thiers B. 2014. Index Herbariorum. A global directory of public herbaria and associated staff. http://sciweb.nybg.org/science2/IndexHerbariorum.asp. 8 Apr. 2014.

Vicentini A, Anderson WR. 1999. Malpighiaceae. In: Ribeiro JELS, Hopkins MJG, Vicentini A, et al. (eds.) Flora da Reserva Ducke - Guia de Identificação das plantas vasculares de uma floresta de terra firme na Amazônia Central. Manaus, INPA-DFID. p. 505-511.

Weberling F. 1989. Morphology of flowers and inflorescences. Cambridge, University Press. 